\title{
An Efficient Routing Protocol for Connecting Vehicular Networks to the Internet
}

\author{
Omar Alghushairy, Wael Aljohani, and Majdi Alharthi \\ Department of Computer Science \& Engineering, University of Bridgeport, USA
}

\begin{abstract}
Technological advancement has continued to grow exponentially threatens to reach greater heights in the next few years. The internet and the different forms of communication have improved the level of technological advancement. Vehicle Ad Hoc Network (VENET) is a new concept in the technological world that ties to connect inner-vehicle communication through cellular and wireless technology in order to improve safety along the roads. This technology offers a variety of application that facilitates road safety. The internet has grown and is used in most industries and it plays a very crucial role in controlling traffic in the busy city centers. With this technology, the drivers expect to face a number of obstacles and limitations and therefore the objective of the paper if to discuss the various challenges faced with the use of the different types of advanced technologies.
\end{abstract}

\section{Keywords: Vehicle AdHoc Network (VENET)}

\section{INTRODUCTION}

Vehicle communication networks have become a very important component for enhancing communication among the drivers. This type of communication cans either be vehicle to vehicle or vehicle to infrastructure. The communication depends on the automated wireless systems of communication also known as the Vehicle Ad Hoc Networks. These networks intend to simplify the connections among a group of vehicles and infrastructure. The use of external networks like the internet is also important in the enhancement of efficiency of such communications [4]. For instance, an external network like the internet can be combined with equipment like wireless cellular networks in order to enhance the effectiveness of vehicle-to-vehicle communication. The recent improvements in terms of cellular technology as well as the increasing interest in the use of the internet have encouraged the exploration of Mobile Ad Hoc Networks whereby the mobile phone devices are used to connect the various drivers. This in turn eases the vehicle-to-vehicle and vehicle-to-infrastructure communications. Below is a diagram to show the connectivity

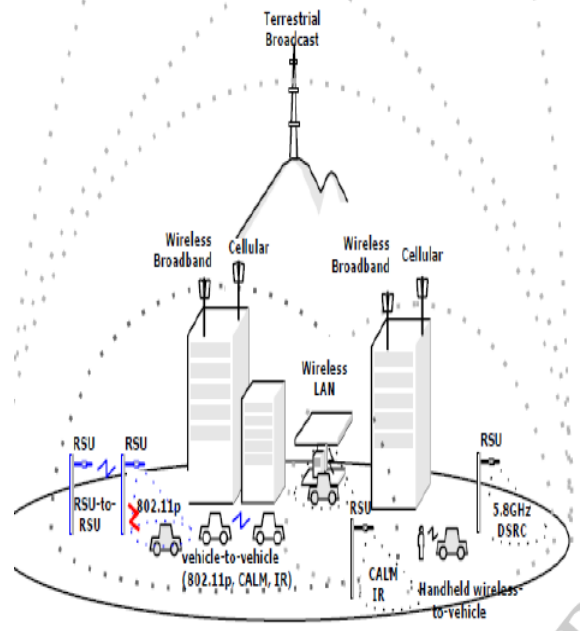

Figure (1).
Communications through networks is a very important part of human life, with all the activities engaged in having to depend on these networks. These networks are found in the individual's homes, offices, schools as well as other places that they frequent.

A majority of the car companies have settled for the use of vehicle communication networks to control the movement of the vehicles and at the same time improve the internal communications. However, in spite of all this, the many explorations into the methods of vehicle communications, the field has had a number of shortcomings and limitations. The biggest issue has been controlling, building and managing the vehicle networks. This paper therefore discusses the problems with more details and provides recommendations on how to solve these problems with the aim of enhancing effectiveness and efficiency in the communication networks [1].

\section{PROBLEM STATEMENT}

The major obstacle to vehicle communication is the inconsistences in the environments and the geographical topologies. This particular issue is more pressing in the rural areas where there is an unfavorable terrain. Features like mountains lower the node densities and thereby reduce the efficiency of communication. The rural areas have less access to the wireless coverage as well as the fixe structures of communication infrastructure thereby limiting the use of wireless connectivity. Therefore, it is quite hard to implement as well as to execute the vehicle communication in the areas [5].

Rural areas experience massive disconnections because of the highly dynamic topology of Vehicular Ad Hoc Networks. Whereas the vehicles in the sparse areas exchange information, disconnections may occur thereby hindering the communications. Just like the rural areas, the urban areas are also characterized with prebuilt highways as well as buildings that hinder vehicular communication. Such obstacles reduce the general potential of the nodes to 
identify and recognize each other thereby hindering the transfer of information. As a result, various hops in communication take place, as immediate neighbors cannot send information efficiently to one another thereby causing delays and in some cases causing an increased number of elapsed recurrent hops.

\section{RELATED WORK}

Vehicular Ad Hoc Networks consist mainly of two types of routing protocol, which include proactive and reactive protocols. Proactive protocols send control packets within the network to update the routing tables. The dynamics of these proactive networks has increased, resulting to network congestion. When the sender demands discovery of routes, the reactive protocols send the control packets. The frequent sending of the control packets by the proactive and reactive protocols overwhelms the Vehicular Ad Hoc Network systems thereby degrading the entire system. The movement of the nodes increases the packet delay and congests the network [1].

Vehicular Ad Hoc Networks also face the problem of mobility. When the nodes move at a very high speed, the network performance is degraded. Due to the uncertainty of the positions of these nodes, the routing protocols are therefore faced to change their routing schedule for efficient delivery of packets to the particular node. Failure to change these routing schedules results in a strenuous delivery of the packets. Moreover, because the topology of the network and the nodes keep fluctuating, it becomes quite difficult to the systems to keep up with the transitions thereby bringing the entire network down. Mobility can also result in routing loops where the packets are routed in a much border manner [6].

For efficient functioning of the Vehicular Ad Hoc Networks system, power is very essential. The mobile network nodes need a frequent and efficient supply of power. In most case, it uses a battery. The battery provides a limited supply of power that cannot sustain an efficient routing of the packets. The power that the battery supplies are therefore is sufficient to deal with the changes in the nature of the topology of the network [3].

\section{PROPOSED SOLUTION AND MATHEMATICAL MODEL SOLUTION}

For individuals to reduce or limit the negative effects and the shortcomings of inter-vehicle communication there has been an improvising of topography based routing models. These include protocols like there active and the proactive routing necessary for packet forwarding. The proactive routing is table driven and therefore designed distinctively to route the information like the forwarding hops that follow are maintained no matter the communication requests. This in turn ensures that the control packets are broadcasted constantly among the various nodes to maintain the links between the nodes. In this way, the flooded paths can be maintained since the control packets can be broadcasted on the nodes that have never been in use. This routing type has numerous advantages considering that it does not require route discovery. Proactive routing therefore enables the communication from the routes to the destination through the maintenance of the control packets in the background [2].

Reactive routing opens a route of communication only when it is essential for nodes to communicate to each other. The only routes maintained are those currently in use. This feature is very important since it reduces the burden upon the network. Once a route is found, a full phase is completed. Geographical routing where the decision to forward is made is based on the position of the destination of a packet and the position of the node's one hop neighbor. Geographical routing assumes that every node knows its location. Moreover, it assumes the sounds responsible for sending information know the location of the receiving nodes by the use of Global Positioning Systems. Graphical routing on the other hand is more vigorous in the Vehicular ad hoc Networks field. Unlike the proactive and reactive routing, it does not exchange the link state information. Moreover, it does not maintain the established routes in the background as in the case of topology based routings. This in turn makes it even more robust [7].

Position based routing makes the routing decisions based on the geographical position of the nodes. Therefore, it is a much better choice in the cases in which mobility poses an issue. The position-based topologies do not need the knowledge of the network topology. Every node is normally aware of its positions as well as the positions of the neighbors. The nodes in this routing type make the decisions thus increasing the efficiency as well as the accuracy of sending the information to the intended and the correct destinations.

Simulation Results or Numerical Results

TABLE 1: SIMULATION PARAMETERS

\begin{tabular}{|c|c|}
\hline Parameters & Value \\
\hline Total simulation time & 2 hours \\
\hline Total number of nodes & 1400 \\
\hline $\begin{array}{l}\text { Approximate total physical road } \\
\text { length after linearization }\end{array}$ & $\begin{array}{c}194.36 \\
\text { miles }\end{array}$ \\
\hline $\begin{array}{l}\text { Average distance between } \\
\text { neighboring vehicles }\end{array}$ & $\begin{array}{l}223.4 \\
\text { meters }\end{array}$ \\
\hline Transmission range & $\begin{array}{c}100 \sim 500 \\
\text { meters }\end{array}$ \\
\hline Movement speed & $\begin{array}{c}12.1 \sim 14.7 \\
\mathrm{~m} / \mathrm{s}\end{array}$ \\
\hline
\end{tabular}

Simulation results

TABLE 2: SIMULATION RESULTS

\begin{tabular}{|c|c|c|c|}
\hline \multirow{2}{*}{$\begin{array}{c}\text { Radio } \\
\text { range }(\mathrm{R}) \\
(\mathrm{m})\end{array}$} & \multicolumn{3}{|c|}{ Transit time $\left(\mathrm{T}_{\text {trans }}\right)$} \\
\cline { 2 - 4 } & Avg $(\mathrm{s})$ & $\begin{array}{c}\text { Max } \\
(\mathrm{s})\end{array}$ & $\begin{array}{c}\text { Standard } \\
\text { deviation }(\mathrm{s})\end{array}$ \\
\hline 100 & 5463.0 & 7077.3 & 1103.7 \\
\hline 200 & 4968.2 & 6692.0 & 847.3 \\
\hline 300 & 0 & 0 & 0 \\
\hline$>=400$ & 0 & 0 & 0 \\
\hline
\end{tabular}

From the above results, the mobility model described by the GTI introduces a random nature for the initial node distribution, the speed of the node and the chosen direction, and the generated trajectories from individual 
models. The table above shows the summary of the simulation results.

According to the results, average-traffic load hours with a radio range less than 200 meters partially connects mobile ad hoc network. At the radio range given, the record shows that the range is less than the standard distance recorded between two vehicles. At this range, the delivery of the message is dependent on the moving mobile nodes rather than forwarding from the intermediate nodes using hop by hop. Thus, the average time for the transit of 5000 seconds is close to the time a vehicle is required to move from the position of the Event node to the position of the End node.

\section{CONCLUSION}

Vehicular Ad Hoc Network systems are very important transportation technologies. They ease the process of controlling the vehicles within a given location. Various transportation companies like the commercial transportation vehicles and the taxis can therefore use the Vehicular Ad Hoc Networks to monitor the activities of all their drivers. Its effectiveness however is limited by factors like power, mobility as well as other environmental factors. Various solutions have been improvised to reduce and limit the shortcomings like the use of position base routing as well as topography routings.

\section{REFERENCES}

11] A. Benslimane, B. Saman and A. Chadi, "An efficient routing protocol for connecting vehicular networks to the Internet," Pervasive and Mobile Computing, Vol. 7 (1). pp. 98 - 113, 2011

[2] M. Zhang and R. S. Wolff, "Routing Protocols for Vehicular Ad Hoc Networks in Rural Areas, ” 2012

[3] J. Tian, L. Han, K. Rothermel and C. Cseh, "Spatially Aware Packet Routing for Mobile Ad hoc Inter- vehicle Radio Networks" In Proceedings of the IEEE Intelligent Transportation Systems, Vol: 2, pp. 1546-1551, 2003

[4] J. Zhao and G. Cao, "VADD: Vehicle-Assisted Data Delivery in Vehicular Ad Hoc Networks, $25^{\text {th }}$ IEEE International Conference on Computer Communications.

[5] Proceedings" Survey of Routing Protocols in Vehicular Ad Hoc Networks.2004 C. Kevin, L. Uichin and M. Gerla, "Advances in Vehicular Ad-Hoc Networks: Developments and Challenges," IGI Global.2009 . U. Lee, R. Cheung and M. Gerla, "Emerging Vehicular Applications, " Chapter 9 of Vehicular

[6] Networks: From Theory to Practice. Chapman \& Hall/Crc Computer and Information Science Series. 2009

S. Jaap, M. Bechler and L. Wolf. "Evaluation of Routing Protocols for Vehicular Ad Hoc Networks in City

[7] Traffic Scenarios. " Proceedings of the 5th International Conference on Intelligent Transportation Systems (ITS) Telecommunications. 2005 